\title{
DETERMINATION OF SPATIAL GRAIN SIZE DISTRIBUTION OF A SINTERED METAL
}

\author{
HENRY AMANKWAH, SIGRID LAUER, SURESHAN KARICHERY AND MD. ASHRAF-UL-ALAM
}

(Received 24, April 2008; Revision Accepted 12, January 2009)

\begin{abstract}
The determination of the spatial grain size distribution of a sintered metal from the size distribution estimated from a sample obtained in the section plane is a stereological problem. This problem is discussed with reference to homothetic particles (cubes of two different sizes) and to a system of three types of grains (fine and coarse cubes and coarse triangular prism). Two models are developed to solve the problem, one taking into account the size of the grains and section profiles only and one that includes shape considerations. The models are tested with simple and artificial examples, as well as with simulated data.
\end{abstract}

KEYWORDS: Sintered metal, fine grain, coarse grain, grain size, section profile

\section{INTRODUCTION}

Sintered metals are very useful for many practical purposes, for example in the top of drills and saws to cut stoneware. Sintering, in general, is the formation conglomerate from different materials to form a new material with different properties. One goal might be to increase the density and strength of the material. It is important to know the distribution of grains in the sintered metal because the properties of the sintered metal largely depend on it.

The purpose of this study is to determine the spatial grain size distribution of a sintered metal. Since it is not possible to determine the distribution in the volume, it is usually estimated by examining a number of cross sections. This way of reasoning is called stereology. Imagine a sintered metal tube which consists of grains of different sizes and shapes. Assume that this tube is cut arbitrarily and that on the cross-section, well-shaped domains (section profiles) can be distinguished with a microscope (see Figure 1.1). The two-dimensional section profiles vary in size and shape and from this information one can estimate the grain size distribution in the volume.

Consider a situation where the sintered metal consists of two kinds of grains - fine and coarse. Let all the fine grains be cubed shaped with diameter $0.75 \mu \mathrm{m}$, and let the coarse grains be either cubes or triangular prisms with dimensions $4 \times 2 \mu \mathrm{m}$. By diameter, we mean the largest distance of all the cross-sections. The problem is approached by determining the section profile distribution in a cross-section and relating it to possible grain distributions in the volume.

The main difficulty is that the ratio of small and large section profiles in the area might differ from the ratio of fine and coarse grains in the volume because coarse grains might appear as small section profiles after cutting. In this case, the probabilities of obtaining a certain section profile when cutting a certain type of grains will play a crucial role.

In this paper, we develop a linear model to compare the quantities on a given cross-section to the quantities in a given volume. At first, two different types of grains (coarse and fine cubes) are considered. Secondly, the model is extended for three types of grains including coarse triangular prisms. The model is then checked with a simple and artificial example, as well as with simulated data.

\section{MODELLING THE PROBLEM}

This work is based on the assumptions of homogeneity and isotropy in the volume. Homogeneity in the volume means that the ratio of the volume occupied by fine grains to that occupied by coarse grains is constant in every control volume that is large compared to the grain size. Isotropy means that the grains do not have any preferred orientation. These assumptions are absolutely necessary to ensure that the information on the cross-section is meaningful.

Henry Amankwah, Department of Mathematics \& Statistics, University of Cape Coast, Cape Coast, Ghana

Sigrid Lauer, Department of Mathematics, University of Kaiserslautern, Germany

Sureshan Karichery, Department of Mathematics, University of Kaiserslautern, Germany

Md. Ashraf-UI-Alam, Department of Mathematics, University of Kaiserslautern, Germany 


\subsection{NOTATIONS 1}

- $\quad \mathrm{U}$ : Typical size of a grain in the volume (e.g. maximal diameter, edge length)

- S: Size of a section profile in the area (maximal diameter)

- $\mathrm{d}$ : Critical size to distinguish between fine and coarse grains respectively between small and large section profiles

- $\vartheta_{F}$ : Intensity of fine grains, i.e. the mean number of grains with size $U \leq d$ per unit volume

- $\vartheta_{C}$ : Intensity of coarse grains, i.e. the mean number of grains with size $U>d$ per unit volume

- $\theta_{F}:$ Planar intensity of fine grains, i.e. the mean number of section profiles per unit area which stem from grains of size $U \leq d$, i.e. $\theta_{F}=U$ (fine grains) $\times \vartheta_{\mathrm{F}}$

- $\theta_{C}$ : Planar intensity of coarse grains, i.e. the mean number of section profiles per unit area which stem from grains of size $U>d$, i.e. $\theta_{C}=U$ (coarse grains) $\times \vartheta_{C}$

- $y_{S}$ : Intensity of small section profiles, i.e. the mean number of section profiles with size $S \leq d$ per unit area

- $y_{L}:$ Intensity of large section profiles, i.e. the mean number of section profiles with size $S>d$ per unit area

- $\quad p_{S F}:=P(S \leq d \mid U \leq d)$ : Probability that a section profile is small under the condition that the corresponding intersected grain is fine

- $\quad p_{L F}:=P(S>d \mid U \leq d)$ : Probability that a section profile is large under the condition that the corresponding intersected grain is fine

- $\quad P_{S C}:=P(S \leq d \mid U>d)$ : Probability that a section profile is small under the condition that the corresponding intersected grain is coarse

- $P_{L C}:=P(S>d \mid U>d)$ : Probability that a section profile is large under the condition that the corresponding intersected grain is coarse

Any quantity with ' ${ }^{\prime}$ 'denotes an estimate or an approximation for the specific quantity.

\subsection{FIRST MODEL}

To get a first idea of the problem, a two-dimensional case is considered, where a cross-section is a straight line. Here, the idea of classifying the section profiles with respect to size and using probabilities of getting small or large section profiles from a certain particle is developed. It was quickly realized that the extension to the three-dimensional case does not cause many difficulties and that was why the description of the first model was started directly with the three-dimensional case.

For simplification in the first model, a distinction between only fine and only coarse grains was looked at, with the intensities $\vartheta_{F}$ and $\vartheta_{C}$ respectively. Also, the section profiles on the cross-section were classified with respect to size only, which gives the intensities $y_{S}$ and $y_{L}$ of small and large section profiles respectively.

A section profile is considered to be small when its maximum diameter $S$ is smaller than or equal to $d$, which is the maximum diameter of a fine grain. Instead of the intensity vector $\left(\vartheta_{F} \vartheta_{C}\right)$ of which the components are respectively the mean number of fine and the mean number of coarse grains per unit volume, the planar intensity vector $\left(\theta_{F}, \theta_{C}\right)$ which is easier to handle because it has the same dimensions as the intensities of the section profiles $\left(y_{S}, y_{L}\right)$ was used.

In the most general case of an arbitrary cut through a structure with an isotropic grain distribution, a typical length of a certain type of grain is its rotation average $\bar{b}$ or the mean breadth

$$
\bar{b}=\frac{1}{4 \pi} \sum_{i=1}^{k} l_{i} \gamma_{i}
$$

where $k$ is the number of edges, $l_{i}$ the length of the ith edge and $\gamma_{i}$ the angle between the surface normal of the two faces touching the $i$ th edge. For example, for a cube of edge length $I, \bar{b}=3 / 2 l$. As a motivation for the definition of 
$\theta$, one can think of a cube with edge length 1 (see Figure 2.1), which is discretized in $N^{3}$ cubic elements. Each element comprises of an arbitrary oriented smaller cube of the same size, in which case the number of grains in the unit volume $\vartheta=N^{3}$. In one layer of unit area with thickness $1 / N$ there are $N^{2}$ grains. The number of section profiles in a cut through one layer also depends on the ratio between the mean breadth $\bar{b}$ of the particles and the layer thickness $1 / N$ (certainly the discretization has to fulfill $1 / N \geq \bar{b}$ ) because the cut might not go through a grain. This yields

$$
\theta=\frac{\bar{b}}{1 / N} N^{2}=\bar{b} N^{3}=\bar{b} \vartheta
$$

As already mentioned this is the way to compute the planar intensity in the most general isotropic case. All the following examples are special non-isotropic cases, the typical length has been calibrated to other quantities (edge length, diameter).

The large section profiles stem certainly from coarse grains, but the small section profile may be part of either a fine or a coarse particle. That is the reason why the intensity of small section profiles $y_{S}$ is the sum of the planar intensity of fine grains $\theta_{F}$, multiplied by the probability $P_{S F}$ to get a small section profile from a fine grain, plus the planar intensity of the coarse grains $\theta_{C}$, multiplied by the probability $P_{S C}$ to cut a coarse grain in such a way that the section profile is small. Likewise, the equation for $y_{L}$ is obtained. Thus we have

$$
\begin{aligned}
& y_{S}=p_{S F} \theta_{F}+p_{S C} \theta_{C}, \\
& y_{L}=p_{L F} \theta_{F}+p_{L C} \theta_{C} .
\end{aligned}
$$

This system of two linear equations can be written as a vector on the left hand side, and a matrix-vector multiplication on the right hand side, hence

$$
\left(\begin{array}{l}
y_{S} \\
y_{L}
\end{array}\right)=\left(\begin{array}{ll}
p_{S F} & p_{S C} \\
p_{L F} & p_{L C}
\end{array}\right)\left(\begin{array}{l}
\theta_{F} \\
\theta_{C}
\end{array}\right)
$$

or

$$
\mathbf{y}=\mathbf{P} \theta
$$

The sum of each column of the above matrix sums up to one: certainly, the probability $p_{L F}$ to cut a fine grain in such a way that the section profile is large equals zero, whereas the probability $p_{S F}$, to cut a fine grain so that the section profile is small equals one. Similarly, in the second column, the probabilities to cut a coarse grain in such a way that the section profile is either small $p_{S C}$ or large $p_{L C}$ sum up to one, that is $p_{S C}+p_{L C}=1$. Such a matrix is known as a probability matrix. For a general description of the continuous stereological problem see Nippe and Ohser (1997) and Nippe and Ohser (1999).

Nowadays, there are techniques to measure the quantities $y_{S}$ and $y_{L}$ in the area. The by far more difficult task to determine the probabilities can be approached by computer simulation (see Section 3 ). If the probability matrix is invertible, we can compute estimates for the unknown quantities in the volume.

\section{Example 1}

As an illustration of the first model, let us consider a very simple, but non-isotropic example, where a structure consisting only of fine and coarse cubes (where the diameter $D$ of the coarse cubes is approximately three times that of the fine cubes, $d$ i.e. $D \approx 3 d$ and the volume of the structure is 8 cubic units) is cut vertically. In this case, it is convenient to calculate the planar intensities as the product of the edge lengths ( 1 for the coarse cube and $1 / 3$ for the fine cube) and the intensities 
$\left(\begin{array}{c}\vartheta_{F} \\ \vartheta_{C}\end{array}\right)=\frac{1}{8}\left(\begin{array}{c}4 \cdot 27 \\ 4\end{array}\right)$,

thus

$$
\left(\begin{array}{c}
\theta_{F} \\
\theta_{C}
\end{array}\right)=\frac{1}{8}\left(\begin{array}{c}
4 \cdot 9 \\
4
\end{array}\right) .
$$

From the section profiles on the cross-section, we can compute the intensities

$$
\left(\begin{array}{l}
y_{S} \\
y_{L}
\end{array}\right)=\frac{1}{4}\left(\begin{array}{c}
2 \cdot 9 \\
2
\end{array}\right) .
$$

Since it is impossible to obtain a large section profile from a fine grain, $P_{L F}=0$ whereas $P_{S F}=1$. Because the structure is cut vertically, it is not possible to get a small section profile which stems from a coarse grain. So $P_{S C}=0$ and $P_{L C}=1$. Hence, the equations for the estimates $\left(\hat{\theta}_{F}, \hat{\theta}_{C}\right)$ of the unknown quantities $\left(\theta_{F}, \theta_{C}\right)$ read as follows:

$$
\left(\begin{array}{l}
y_{S} \\
y_{L}
\end{array}\right)=\left(\begin{array}{ll}
1 & 0 \\
0 & 1
\end{array}\right)\left(\begin{array}{l}
\hat{\theta}_{F} \\
\theta_{C}
\end{array}\right) .
$$

One immediately sees that the estimates are in fact exact in this case.

\subsection{NOTATIONS 2}

In addition to the notations introduced before, we need the following:

- $\vartheta_{C}^{c}$ : Intensity of coarse cubes, i.e. the mean number of cubes with size $U>d$ per unit volume

- $\vartheta_{C}^{t p}$ : Intensity of coarse triangular prisms, i.e. the mean number of triangular prisms with size $U>d$ per unit volume

- $\quad \theta_{C}^{c}$ : Planar intensity of coarse cubes, i.e. the mean number of section profiles per unit area which stem from cubes with size $U>d$, i.e. $\theta_{C}=U($ coarse cube $) \times \vartheta_{C}$

- $\quad \theta_{C}^{t p}$ : Planar intensity of coarse triangular prisms, i.e. the mean number of section profiles per unit area which stem from triangular prisms with size $U>d$, i.e. $\theta_{C}=U$ (coarse triangular prism) $\times \vartheta_{C}$

- $\quad y_{S n}$ : Intensity of small $n$-noded section profiles, i.e. the mean number of $n$-noded section profiles with size $S \leq d$ per unit area, $n=3,4,5,6$

- $\quad y_{L n}$ : Intensity of large $n$-noded section profiles, i.e. the mean number of $n$-noded section profiles with size $S>d$ per unit area, $n=3,4,5,6$

- $\quad P_{S F_{n}}:=\mathrm{P}(S \leq d, n$ corner nodes $\mid U \leq d)$ : Probability that a section profile is small and has $n$ corner nodes under the condition that the corresponding intersected grain is fine.

- $\quad P_{L F_{n}}:=P(S>d, n$ corner nodes $\mid U \leq d)$ : Probability that a section profile is large and has $n$ corner nodes under the condition that the corresponding intersected grain is fine

- $\quad P_{S C_{n}}^{c}:=P(S \leq d, n$ corner nodes $\mid U($ cube $)>d)$ :Probability that a section profile is small and has $n$ corner nodes under the condition that the corresponding intersected grain is a coarse cube

- $\quad P_{L C_{n}}^{c}:=P(S>d, n$ corner nodes $\mid U($ cube $)>d)$ : Probability that a section profile is large and has $n$ corner nodes under the condition that the corresponding intersected grain is a coarse cube

- $\quad P_{S C n}^{t p}:=P(S \leq d, n$ corner nodes $\mid U($ triangular prism $)>d)$ : Probability that a section profile is small and has $n$ corner nodes under the condition that the corresponding intersected grain is a coarse triangular prism 
- $\quad P_{L C n}^{t p}:=P(S>d, n$ corner nodes $\mid U($ triangular prism $)>d)$ : Probability that a section profile is large and has $n$ corner nodes under the condition that the corresponding intersected grain is a coarse triangular prism

Any quantity with a ' $\wedge$ ' denotes an estimate or an approximation for the specific quantity.

\subsection{SECOND MODEL}

The second model is an extension of the first model and not only size classifies a particle or a section profile but also the shape. Hence, the planar intensity vector is extended by a third component. To be precise, the planar intensity of the coarse grains is divided into $\theta_{C}^{c}$ for coarse cubes and $\theta_{C}^{t p}$ for triangular prisms.

Cutting a triangular prism arbitrarily may result in section profiles with 3 to 5 nodes, the cubes can produce additionally six-noded section profiles. Classifying the section profiles with respect to size and shape results in the intensities of small and large $n$-noded section profiles $y_{S n}$ and $y_{L n}$, where $n \in\{3,4,5,6\}$. Also, the probability matrix has to be extended. First of all it has a third column for the probabilities to get certain section profiles from the triangular prism and secondly it has eight rows now. On each row, there are probabilities to get one of the eight types of section profiles from the three types of grains.

$$
\left(\begin{array}{l}
\mathrm{y}_{S 3} \\
y_{S 4} \\
y_{S 5} \\
y_{S 6} \\
y_{L 3} \\
y_{L 4} \\
y_{L 5} \\
y_{L 6}
\end{array}\right)=\left(\begin{array}{lll}
p_{S F 3} & p_{S C 3}^{c} & p_{S C 3}^{t p} \\
p_{S F 4} & p_{S C 4}^{c} & p_{S C 4}^{t p} \\
p_{S F 5} & p_{S C 5}^{c} & p_{S C 5}^{t p} \\
p_{S F 6} & p_{S C 6}^{c} & p_{S C 6}^{t p} \\
p_{L F 3} & p_{L C 3}^{c} & p_{L C 3}^{t p} \\
p_{L F 4} & p_{L C 4}^{c} & p_{L C 4}^{t p} \\
p_{L F 5} & p_{L C 5}^{c} & p_{L C 5}^{t p} \\
p_{L F 6} & p_{L C 6}^{c} & p_{L C 6}^{t p}
\end{array}\right) \quad\left(\begin{array}{l}
\theta_{F} \\
\\
\theta_{C}^{c} \\
\\
\theta_{C}^{t p}
\end{array}\right)
$$

To solve this over-determined system of equations one needs to use a generalized inverse.

\section{Example 2}

To illustrate the second model, a very simple artificial example as shown in Figure 2.3 is considered. In this structure of volume 27 cubic units (see Figure 2.3 a), a cube with diameter $D$ is either occupied by one coarse cube or by twenty-seven fine cubes or by two coarse triangular prisms and the intensities take the values

$$
\left(\begin{array}{c}
\vartheta_{F} \\
\vartheta_{C}^{c} \\
\vartheta_{C}^{t p}
\end{array}\right)=\frac{1}{27}\left(\begin{array}{c}
9 \cdot 27 \\
9 \\
9 \cdot 2
\end{array}\right)=\left(\begin{array}{c}
3 \\
\frac{1}{3} \\
\frac{2}{3}
\end{array}\right) .
$$

After multiplying each component with the edge length of the corresponding grain, we get

$$
\left(\begin{array}{c}
\theta_{F} \\
\theta_{C}^{c} \\
\theta_{C}^{t p}
\end{array}\right)=\frac{1}{27}\left(\begin{array}{c}
9 \cdot 9 \\
9 \\
9 \cdot 2
\end{array}\right)=\left(\begin{array}{c}
3 \\
\frac{1}{3} \\
\frac{2}{3}
\end{array}\right) .
$$

After a vertical cut, one only observes four-noded small section profiles with the intensity $y_{S 4}=(3 \cdot 9) / 9$. The large section profiles are either triangles or squares with the intensities $y_{L 3}=(3 \cdot 2) / 9$ and $y_{L 4}=3 / 9$ respectively. Because in this case of vertical cutting, the fine cubes can produce only small four-noded section profiles, the only probability in the first column of the probability matrix that is non-zero is $P_{S F 4}$ and thus $P_{S F 4}=1$. Likewise, the only type of section profile we get from the coarse cubes is a large square, so $p_{L C 4}^{c}=1$ and the only type of section profile from the triangular prisms is a large triangle, so $p_{L C 3}^{t p}=1$. All the other entries in the probability matrix are zeros. Hence the equations for the estimates of the planar intensities read 


$$
\frac{1}{9}\left(\begin{array}{c}
0 \\
3 \cdot 9 \\
0 \\
0 \\
3 \cdot 2 \\
3 \\
0 \\
0
\end{array}\right)=\left(\begin{array}{lll}
0 & 0 & 0 \\
1 & 0 & 0 \\
0 & 0 & 0 \\
0 & 0 & 0 \\
0 & 0 & 1 \\
0 & 1 & 0 \\
0 & 0 & 0 \\
0 & 0 & 0
\end{array}\right)\left(\begin{array}{c}
\hat{\theta}_{\mathrm{F}} \\
\\
\hat{\theta}_{C}^{c} \\
\\
\hat{\theta}_{C}^{t p}
\end{array}\right)
$$

and one can immediately see that the estimates

$$
\left(\begin{array}{c}
\hat{\theta}_{F} \\
\hat{\theta}_{C}^{c} \\
\hat{\theta}_{C}^{t p}
\end{array}\right)=\frac{1}{9}\left(\begin{array}{c}
3 \cdot 9 \\
3 \\
3 \cdot 2
\end{array}\right)=\left(\begin{array}{c}
3 \\
\frac{1}{3} \\
\frac{2}{3}
\end{array}\right)
$$

equal the real quantities $\left(\theta_{F}, \theta_{C}^{c}, \theta_{C}^{t p}\right)$.

\section{SIMULATION OF THE PROBABILITIES}

The task of determining the probabilities of getting different types of section profiles from a cube or triangular prism was approached by computer simulation. The method used was based on the following steps:

1. Construction of a particle (cube or triangular prism) of size a

2. Random rotation of this particle using "Euler angles"

3. Generation of the section profiles by intersecting the rotated particle with randomly chosen planes

4. Classification of the section profiles with respect to size and shape and storing the information

5. Calculation of the probabilities using the stored information

Steps 2, 3 and 4 are repeated for a large number of times and from the stored information of the section profiles the required probabilities were calculated. The flow chart in Figure 3.1 gives a better idea of the simulation procedure. A more detailed discussion of the steps is presented as follows:

The representation of a particle centered at the origin by its $n$ corner nodes $\left(x_{i}, y_{i}, z_{i}\right), i=1,2,3, \ldots, n$, in Cartesian coordinates in the subroutine of the simulation program is self-explanatory. The coordinates $\left(x_{i}^{\prime}, y_{i}^{\prime}, z_{i}^{\prime}\right)$, $i=1,2,3, \ldots, n$, of the rotated particle by the Euler angles $(\psi, \vartheta, \varphi)$ are obtained from

$$
\left(\begin{array}{l}
x_{i}^{\prime} \\
y_{i}^{\prime} \\
z_{i}^{\prime}
\end{array}\right)=\left(\begin{array}{ccc}
\cos \varphi & \sin \varphi & 0 \\
-\sin \varphi & \cos \varphi & 0 \\
0 & 0 & 1
\end{array}\right)\left(\begin{array}{ccc}
1 & 0 & 0 \\
0 & \cos \vartheta & \sin \vartheta \\
0 & -\sin \vartheta & \cos \vartheta
\end{array}\right)\left(\begin{array}{ccc}
\cos \psi & \sin \psi & 0 \\
-\sin \psi & \cos \psi & 0 \\
0 & 0 & 1
\end{array}\right)\left(\begin{array}{l}
x_{i} \\
y_{i} \\
z_{i}
\end{array}\right)
$$

where the Euler angles are random variables. Multiplying this combination of matrices with a coordinate vector performs first a rotation around the $z$-axis by the angle $\psi$, secondly a rotation around the recently obtained new $x$-axis by the angle $\vartheta$ and finally a rotation around the new $z$-axis by $\varphi$. The requirement for isotropy is fulfilled by using the following conditions:

1. The random angles $\psi, \vartheta, \varphi$ are independent of each other

2. The points $(1, \vartheta, \varphi)$ given in spherical polar coordinates are uniformly distributed on the unit sphere whenever

(a) $\varphi$ is uniformly distributed on the interval $[0,2 \pi)$ and

(b) $\xi=\cos \vartheta$ is uniformly distributed on [-1,1]

3. The angles $\psi$ are uniformly distributed on $[0,2 \pi)$

Such a random rotation of a given particle can be performed by the simulation programs. From each rotated particle a series of section profiles is generated by intersecting it with planes. These planes are parallel to the $x-y$-plane and the $z$-coordinate is chosen randomly in $\left(z_{\min }, z_{\max }\right)$, where $z_{\min }\left(z_{\text {max }}\right)$ is the minimum (maximum) $z$-coordinate of the 
rotated particle. This procedure ensures that a real section profile is generated, whereas the intersection set of an arbitrary plane with a non-rotated particle might be empty.

Cutting a cube (or triangular prism) arbitrarily might result in section profiles with 3 to 6 (3 to 5 ) corner nodes. The number of corner nodes and the size, which is the largest distance between two corner nodes, are stored for each section profile.

The probability of obtaining an $n$-gon (or an $n$ - noded section profile) with size $S$ smaller (or larger) than $d$ (which is the critical size) is the number of that type of section profiles divided by the total number of section profiles that has been classified. Figure 3.2 and Figure 3.3 show the frequency distribution of $n$-gons of a cube and a triangular prism of size 1 , where a total of 100,000 section profiles have been classified. With these simulation results, it is easy to calculate the desired probabilities. For example, the probability of obtaining a triangular section profile which is smaller than 0.3 from the triangular prism is calculated as follows:

One integrates over the * curve from 0 to 0.3 and divides this by the total number of section profiles that have been classified. The following tables show the probabilities of getting an $n$-gon from a cube, respective a regular triangular prism of size 1.

Table 3.1: Probability of getting an $n$-gon with size $S \leq d$ from a cube of size 1 .

\begin{tabular}{|c|l|l|l|l|c|}
\hline$d$ & $n=3$ & $n=4$ & $n=5$ & $n=6$ & $n \in\{3,4,5,6\}$ \\
\hline 0.1 & 0.0382 & 0.0000 & 0.0000 & 0.0000 & 0.0382 \\
\hline 0.2 & 0.0767 & 0.0000 & 0.0000 & 0.0000 & 0.0767 \\
\hline 0.3 & 0.1151 & 0.0000 & 0.0000 & 0.0000 & 0.1151 \\
\hline 0.4 & 0.1535 & 0.0000 & 0.0000 & 0.0000 & 0.1535 \\
\hline 0.5 & 0.1914 & 0.0000 & 0.0000 & 0.0000 & 0.1914 \\
\hline 0.6 & 0.2291 & 0.0276 & 0.0000 & 0.0000 & 0.2567 \\
\hline 0.7 & 0.2531 & 0.1475 & 0.0000 & 0.0000 & 0.4006 \\
\hline 0.8 & 0.2615 & 0.2728 & 0.0000 & 0.0000 & 0.5343 \\
\hline 0.9 & 0.2616 & 0.4815 & 0.1538 & 0.0294 & 0.9264 \\
\hline 1.0 & 0.2616 & 0.5161 & 0.1818 & 0.0404 & 1.0000 \\
\hline
\end{tabular}

Table 3.2: Probability of getting an $n$-gon with size $S \leq d$ from a rectangular prism of size 1 .

\begin{tabular}{|c|l|l|l|c|}
\hline$D$ & $n=3$ & $n=4$ & $n=5$ & $n \in\{3,4,5\}$ \\
\hline 0.1 & 0.0473 & 0.0000 & 0.0000 & 0.0473 \\
\hline 0.2 & 0.0927 & 0.0000 & 0.0000 & 0.0927 \\
\hline 0.3 & 0.1383 & 0.0000 & 0.0000 & 0.1383 \\
\hline 0.4 & 0.1848 & 0.0000 & 0.0000 & 0.1848 \\
\hline 0.5 & 0.2318 & 0.0000 & 0.0000 & 0.2318 \\
\hline 0.6 & 0.2775 & 0.0000 & 0.0000 & 0.2775 \\
\hline 0.7 & 0.3241 & 0.0000 & 0.0000 & 0.3241 \\
\hline 0.8 & 0.4233 & 0.2225 & 0.0000 & 0.6458 \\
\hline 0.9 & 0.4595 & 0.3716 & 0.0224 & 0.8536 \\
\hline 1.0 & 0.4695 & 0.4453 & 0.0852 & 1.0000 \\
\hline
\end{tabular}




\section{EVALUATION OF MODELS USING SIMULATED DATA \\ 4.1 SIMULATION TO GET DATA}

Because of lack of realistic data, a simulation of arbitrary cuts through the two different structures of grains that was carried out. In both structures of Figure 4.1, the intensities of each type of grain, respective planar intensities can be easily computed:
(a) $\left(\begin{array}{l}\vartheta_{F} \\ \vartheta_{C}\end{array}\right)=\frac{1}{64}\left(\begin{array}{c}32 \cdot 27 \\ 32\end{array}\right)$
$\left(\begin{array}{l}\theta_{F} \\ \theta_{C}\end{array}\right)=\frac{\sqrt{3}}{64}\left(\begin{array}{c}32 \cdot 9 \\ 32\end{array}\right)=\left(\begin{array}{l}7.794 \\ 0.866\end{array}\right)$.
(b) $\left(\begin{array}{c}\vartheta_{F} \\ \vartheta_{C}^{c} \\ \vartheta_{C}^{t p}\end{array}\right)=\frac{1}{27}\left(\begin{array}{c}9 \cdot 27 \\ 9 \\ 9 \cdot 2\end{array}\right)$,
$\left(\begin{array}{c}\theta_{F} \\ \theta_{C}^{c} \\ \theta_{C}^{t p}\end{array}\right)=\frac{\sqrt{3}}{27}\left(\begin{array}{c}9 \cdot 9 \\ 9 \\ 9 \cdot 2\end{array}\right)=\left(\begin{array}{c}5.196 \\ 0.577 \\ 1.155\end{array}\right)$.

The structure is rotated randomly and then cut by planes that are parallel to the $x-y-p l a n e$. Each time the total area of intersection and the number of section profiles of each type (classification with respect to size as in first model or with respect to size and shape as in second model with critical size $d=\sqrt{3} / 3 \approx 0.577$ ) are stored. The section profile intensities can be calculated from this information.

\subsection{EVALUATION OF THE MODELS}

Using the simulated probabilities, as explained in the previous section, the two models can be checked Considering the first model on structure (a), then from the simulation,

$$
\hat{\mathbf{y}}=\left(\begin{array}{c}
\hat{y}_{F} \\
\hat{y}_{C}
\end{array}\right)=\left(\begin{array}{l}
8.7644 \\
0.8321
\end{array}\right)
$$

and

$$
\hat{\mathbf{P}}=\left(\begin{array}{ll}
\hat{P}_{S F} & \hat{P}_{S C} \\
\hat{P}_{L F} & \hat{P}_{L C}
\end{array}\right)=\left(\begin{array}{ll}
1 & 0.1281 \\
0 & 0.8719
\end{array}\right) .
$$

Solving the equations

$$
\left(\begin{array}{l}
\hat{y}_{F} \\
\hat{y}_{C}
\end{array}\right)=\left(\begin{array}{ll}
\hat{P}_{S F} & \hat{P}_{S C} \\
\hat{P}_{L F} & \hat{P}_{L C}
\end{array}\right)\left(\begin{array}{l}
\hat{\boldsymbol{\theta}}_{F} \\
\hat{\boldsymbol{\theta}}_{C}
\end{array}\right)
$$

for the estimates of the planar intensities results in

$$
\left(\begin{array}{l}
\hat{\boldsymbol{\theta}}_{F} \\
\hat{\boldsymbol{\theta}}_{C}
\end{array}\right)=\hat{\mathbf{P}}^{-1} \hat{\mathbf{y}}=\left(\begin{array}{l}
8.6421 \\
0.9544
\end{array}\right) .
$$

The relative error is given by

$$
\left(\begin{array}{l}
e_{F} \\
e_{C}
\end{array}\right):=\left(\begin{array}{l}
\frac{\left|\hat{\theta}_{F}-\theta_{F}\right|}{\theta_{F}} \\
\frac{\left|\hat{\theta}_{C}-\theta_{C}\right|}{\theta_{C}}
\end{array}\right)=\left(\begin{array}{l}
11 \% \\
10 \%
\end{array}\right) .
$$

Now, considering the second model on structure (a), from the simulation, 


$$
\hat{\mathbf{y}}=\left(\begin{array}{l}
\hat{y}_{S 3} \\
\hat{y}_{S 4} \\
\hat{y}_{S 5} \\
\hat{y}_{S 6} \\
\hat{y}_{L 3} \\
\hat{y}_{L 4} \\
\hat{y}_{L 5} \\
\hat{y}_{L 6}
\end{array}\right)=\left(\begin{array}{l}
2.7190 \\
3.8985 \\
1.7192 \\
0.4275 \\
0.1490 \\
0.4456 \\
0.1856 \\
0.0515
\end{array}\right)
$$

and

$$
\hat{\mathbf{P}}=\left(\begin{array}{ll}
\hat{P}_{S F 3} & \hat{P}_{S C 3} \\
\hat{P}_{S F 4} & \hat{P}_{S C 4} \\
\hat{P}_{S F 5} & \hat{P}_{S C 5} \\
\hat{P}_{S F 6} & \hat{P}_{S C 6} \\
\hat{P}_{L F 3} & \hat{P}_{L C 3} \\
\hat{P}_{L F 4} & \hat{P}_{L C 4} \\
\hat{P}_{L F 5} & \hat{P}_{L C 5} \\
\hat{P}_{L F 6} & \hat{P}_{L C 6}
\end{array}\right)=\left(\begin{array}{ll}
0.262 & 0.131 \\
0.516 & 0.000 \\
0.182 & 0.000 \\
0.040 & 0.000 \\
0.000 & 0.131 \\
0.000 & 0.516 \\
0.000 & 0.182 \\
0.000 & 0.040
\end{array}\right) .
$$

Solving the equations using Pinv with default settings in MATLAB, gives

$$
\left(\begin{array}{l}
\hat{\boldsymbol{\theta}}_{F} \\
\hat{\boldsymbol{\theta}}_{C}
\end{array}\right)=\hat{\mathbf{P}}^{-\mathbf{1}} \hat{\mathbf{y}}=\left(\begin{array}{l}
8.1627 \\
1.0772
\end{array}\right),
$$

for which the relative error is given by

$$
\left(\begin{array}{l}
e_{F} \\
e_{C}
\end{array}\right)=\left(\begin{array}{c}
4.7 \% \\
24 \%
\end{array}\right)
$$

The second model which uses more detailed information about the section profiles gives much better values for $\hat{\boldsymbol{\theta}}_{F}$. This is clear because looking at the frequency distribution of section profiles on a simulated cross-section in Figure 4.2 and Figure 4.3 and paying attention to the different scaling, one can see that much more information about the fine grains has been used.

Up to this point, the evaluation of the models has been done using the $4 \times 4 \times 4$ structure in Figure 4.1 (a). Simulating cross-sections of a $6 \times 6 \times 6$ structure did not show any significant difference. A simulation of cuts through a larger structure was not possible because of the limit of computer memory.

Considering the second model on structure (b), then from the simulation,

$$
\hat{\mathbf{y}}=\left(\begin{array}{l}
\hat{y}_{S 3} \\
\hat{y}_{S 4} \\
\hat{y}_{S 5} \\
\hat{y}_{S 6} \\
\hat{y}_{L 3} \\
\hat{y}_{L 4} \\
\hat{y}_{L 5} \\
\hat{y}_{L 6}
\end{array}\right)=\left(\begin{array}{l}
2.0415 \\
2.6037 \\
1.1136 \\
0.2514 \\
0.2564 \\
0.5177 \\
0.2540 \\
0.1815
\end{array}\right)
$$


and

$$
\hat{\mathbf{P}}=\left(\begin{array}{lll}
\hat{P}_{S F 3} & \hat{P}_{S C 3}^{c} & \hat{P}_{S C 3}^{t p} \\
\hat{P}_{S F 4} & \hat{P}_{S C 4}^{c} & \hat{P}_{S C 4}^{t p} \\
\hat{P}_{S F 5} & \hat{P}_{S C 5}^{c} & \hat{P}_{S C 5}^{t p} \\
\hat{P}_{S F 6} & \hat{P}_{S C 6}^{c} & \hat{P}_{S C 6}^{t p} \\
\hat{P}_{L F 3} & \hat{P}_{L C 3}^{c} & \hat{P}_{L C 3}^{t p} \\
\hat{P}_{L F 4} & \hat{P}_{L C 4}^{c} & \hat{P}_{L C 4}^{t p} \\
\hat{P}_{L F 5} & \hat{P}_{L C 5}^{c} & \hat{P}_{L C 5}^{t p} \\
\hat{P}_{L F 6} & \hat{P}_{L C 6}^{c} & \hat{P}_{L C 6}^{t p}
\end{array}\right)=\left(\begin{array}{lll}
0.262 & 0.131 & 0.174 \\
0.516 & 0.000 & 0.000 \\
0.182 & 0.000 & 0.000 \\
0.040 & 0.000 & 0.285 \\
0.000 & 0.131 & 0.000 \\
0.000 & 0.516 & 0.451 \\
0.000 & 0.182 & 0.090 \\
0.000 & 0.040 & 0.000
\end{array}\right)
$$

can be calculated.

Solving the system $\hat{\mathbf{y}}=\hat{\mathbf{P}} \hat{\boldsymbol{\theta}}$ for the estimates $\left(\hat{\boldsymbol{\theta}}_{\mathbf{F}}, \hat{\boldsymbol{\theta}}_{\mathbf{C}}^{\mathbf{c}}, \hat{\boldsymbol{\theta}}_{\mathbf{C}}^{\text {tp }}\right)$ results in

$$
\left(\begin{array}{c}
\hat{\boldsymbol{\theta}}_{\mathbf{F}} \\
\hat{\boldsymbol{\theta}}_{\mathbf{C}}^{\mathbf{c}} \\
\hat{\boldsymbol{\theta}}_{\mathbf{C}}^{\mathbf{t p}}
\end{array}\right)=\hat{\mathbf{P}}^{-1} \hat{\mathbf{y}}=\left(\begin{array}{l}
5.5071 \\
0.4370 \\
0.9248
\end{array}\right)
$$

where the relative error is given by

$$
\left(\begin{array}{l}
e_{F} \\
e_{C}^{c} \\
e_{C}^{t p}
\end{array}\right)=\left(\begin{array}{c}
6 \% \\
24 \% \\
19.5 \%
\end{array}\right) .
$$

Unfortunately, the models could not be tested with realistic data; however, with the results from the simulations and the errors in a range below $30 \%$, one can say that the models seem to work quite well for this ill-posed problem. It should be noted however that the examples considered are of non-isotropic grain distributions where the general typical length, the rotation average, $\bar{b}$ has been calibrated to other quantities (edge length, diameter).

\section{CONCLUSION}

It could be said finally that the models do express a relationship between the quantities on the cross-section and the quantities in the volume under the assumptions of homogeneity and isotropy. An extension of the second model to different kinds of grains is possible because every kind of grain is treated separately. It was however not possible to check the models in more detail due to lack of realistic data for the section profiles and the quantities in the volume. Sources of error might be from the use of estimates or approximations. Also the strong homogeneity and anisotropy that might occur in reality will cause problems.

\section{REFERENCES}

Gauthier G. and Co., 1996. Morphological Segmentation of Cutting Tools. Microsc. Micronal. Microstruct. 7. pp 339344.

Gauthier G. and Co., 1994. Segmentation of Grain Boundaries in WC-Co Cermets. Acta Stereol. 13. pp 203-208.

Mehnert K. and Co., 1998. Testing Stereological Methods for the Estimation of Spatial Size Distribution by means of Computer- Simulated Grain Structure. Materials Science \& Engineering A246. pp 207-212.

Nippe, M. and Ohser, J., 1999. The Stereological Unfolding Problem for Systems of Homothetic Particles. Pattern Recognition. 32: 1649-1655.

Ohser, J. and Mucklich, F., 2000. Statistical Analysis of Microstructure in Materials Science. John Wiley and Sons.

Ohser J. and Nippe, M., 1997. Stereology of Cubic Particles: Various Estimators for the Size Distribution. Journal of Microscopy. 187. Pt. 1. pp 22-33.

Voss, K., 1982. Frequencies of n-Polygons in Planar Sections of Polyhedrons. Journal of Microscopy. 128. Pt. 2. pp 111-120. 


\section{APPENDIX}

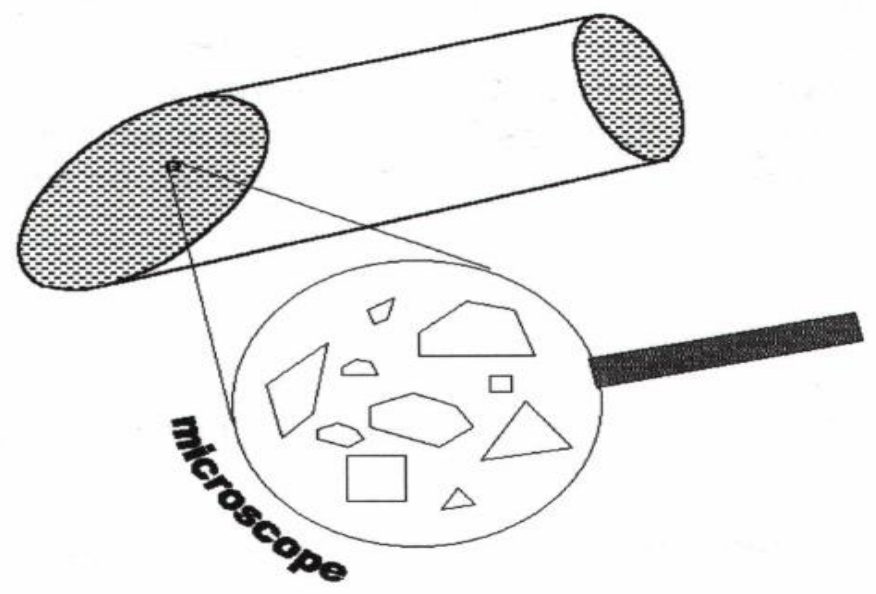

Figure 1.1: Cross-section examination

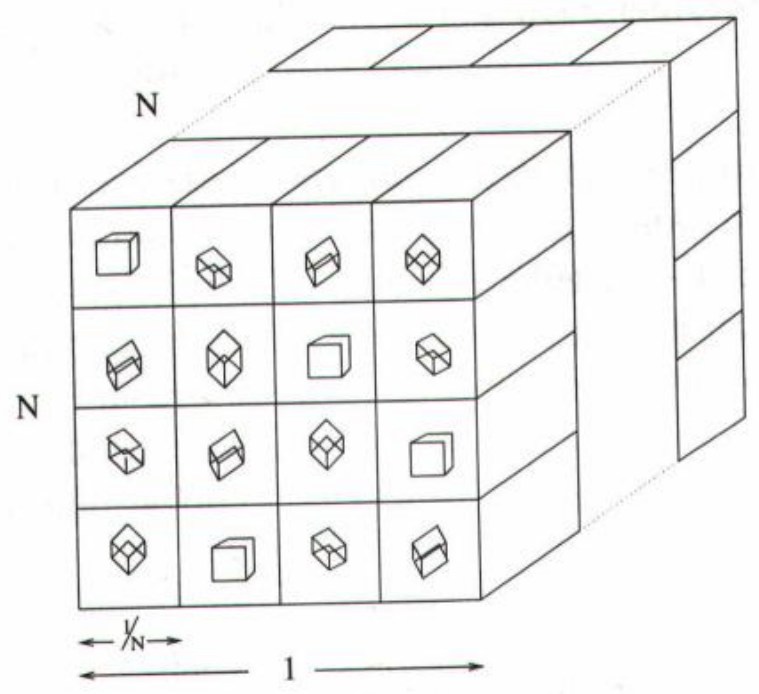

Figure 2.1: Unit volume, discretized into $N^{3}$ cubic elements, each containing an arbitrary oriented cube

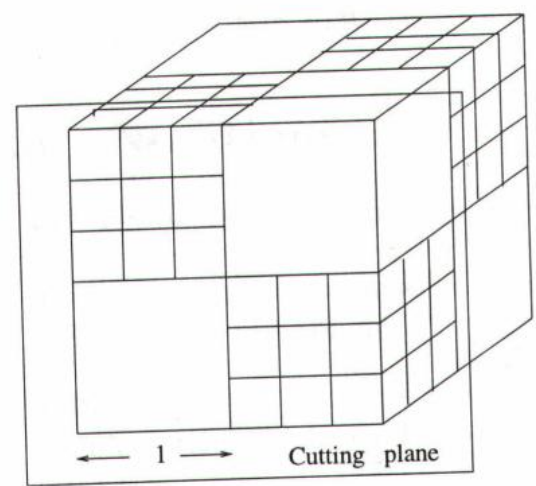

a)
Cross-section

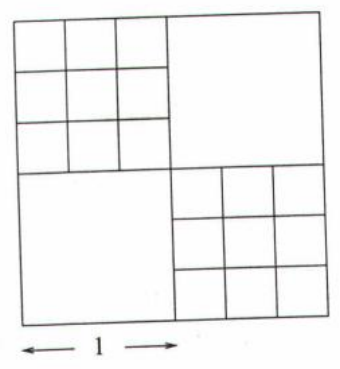

b)

Figure 2.2: (a) A cube of diameter $D=\sqrt{3}$ is occupied by one coarse cube or by 27 fine cubes. (b) A cross-section 


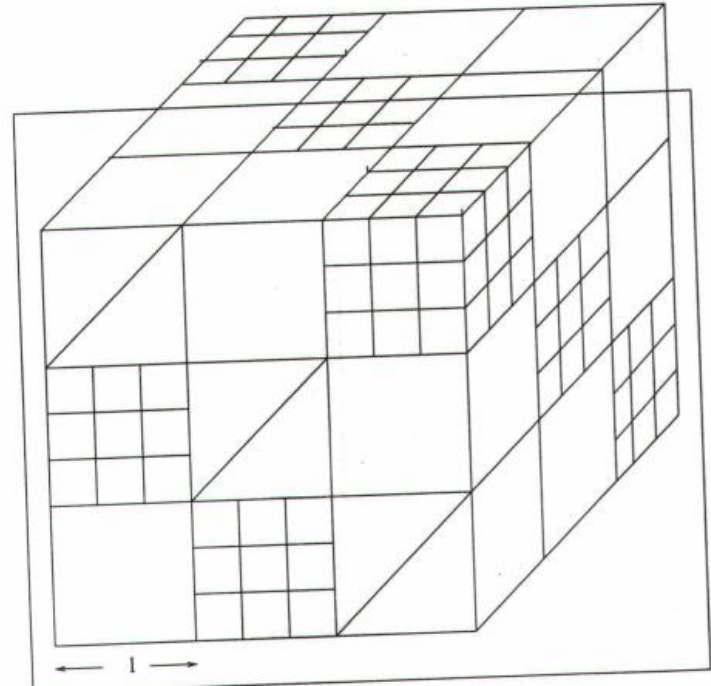

a)

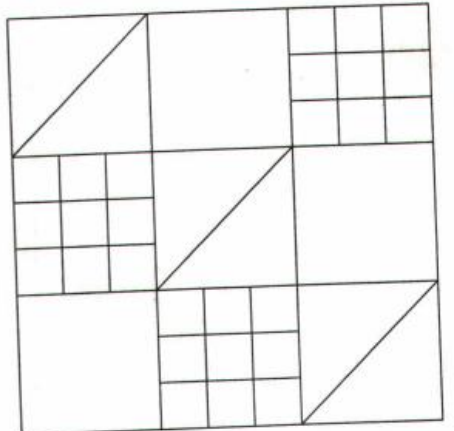

b)

Figure 2.3: (a) A cube of diameter $D=\sqrt{3}$ is either occupied by one coarse cube or by 27 fine cubes or by two triangular prisms.

(b) A cross-section

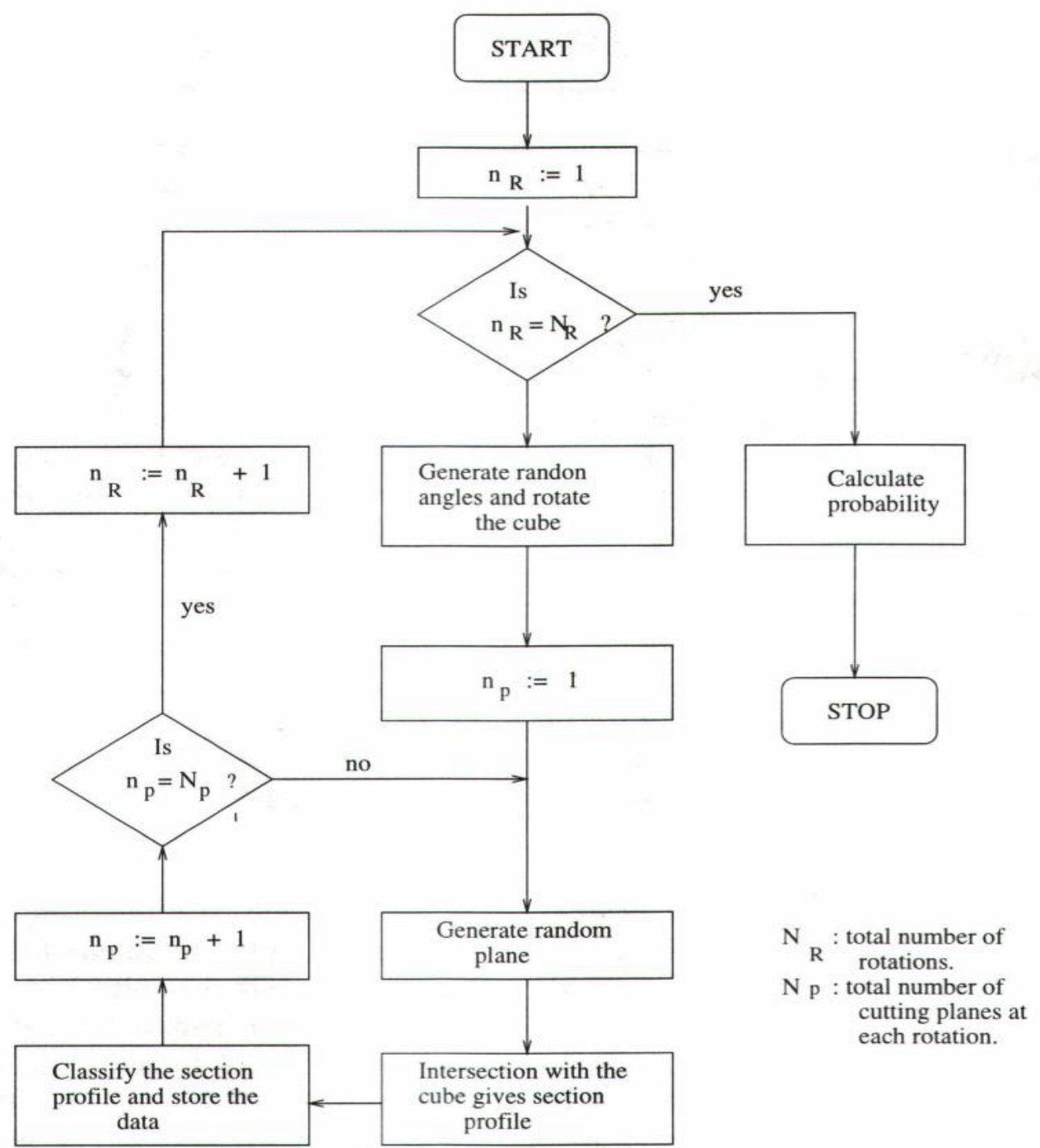

Figure 3.1: A flow chart of the simulation procedure 


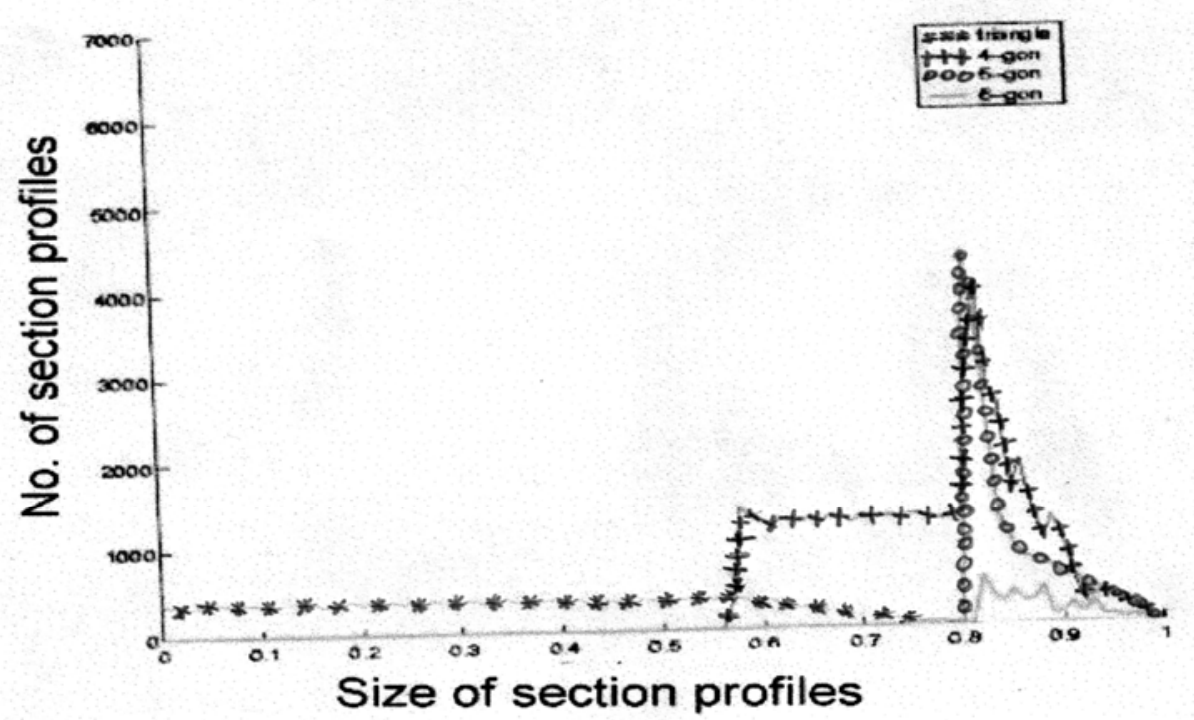

Figure 3.2: A frequency distribution of $n$-gons for a cube of size 1.

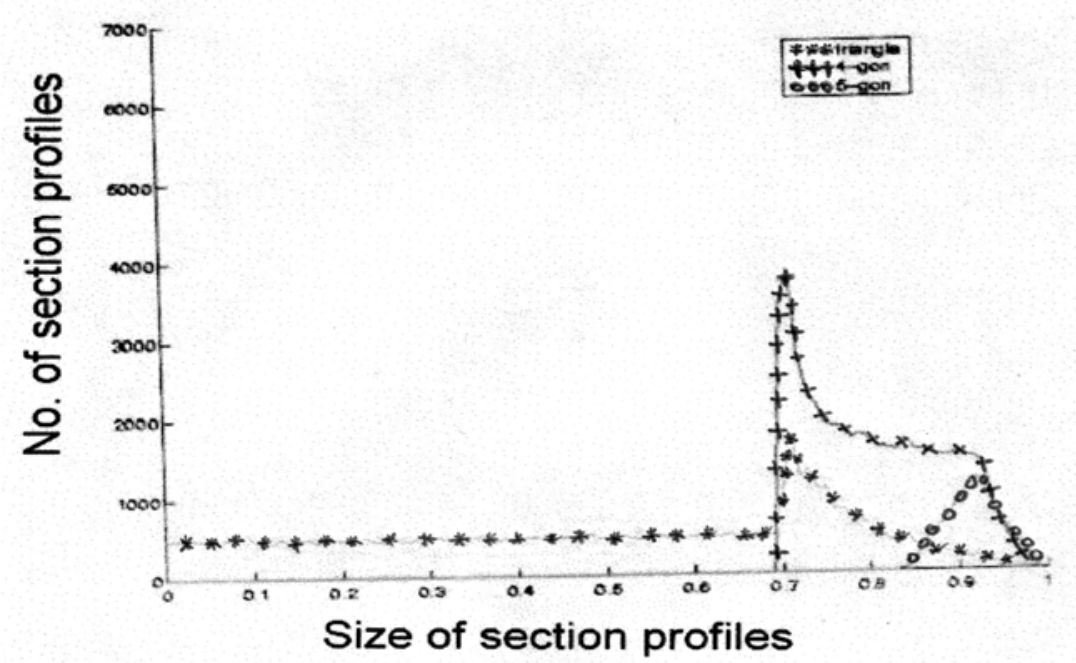

Figure 3.3: A frequency distribution of $n$-gons for a regular triangular prism of size 1 .
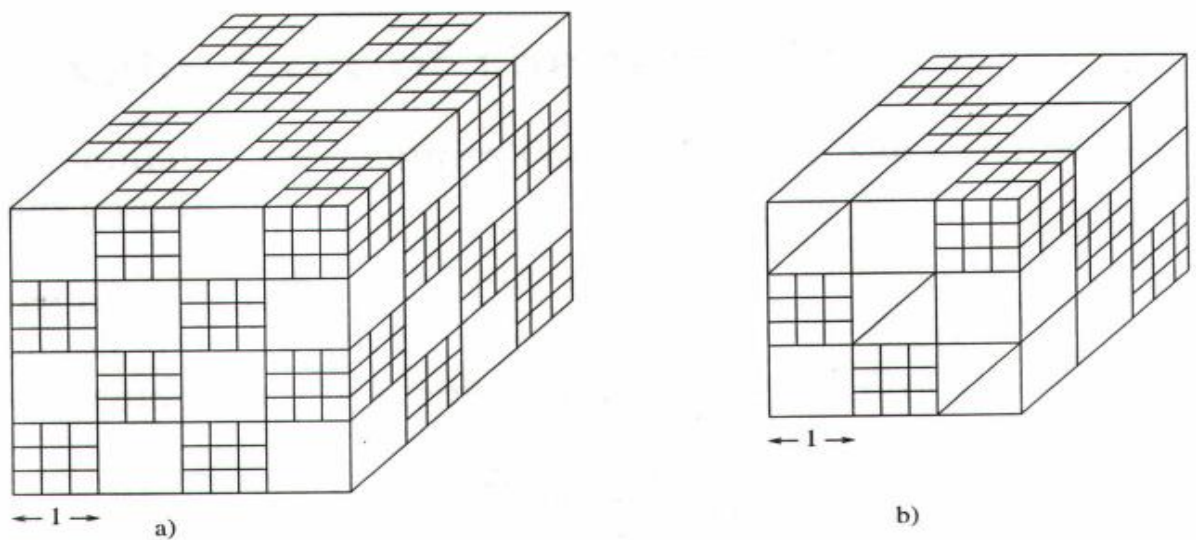

b)

Figure 4.1: (a) A given cube of volume 64 cubic units with a diameter $D=\sqrt{3}$, is either occupied by one coarse cube or by 27 fine cubes.

(b) A given cube of volume 27 cubic units with a diameter

$D=\sqrt{3}$, is either occupied by one coarse cube or by 27 fine cubes or by 2 coarse triangular prisms. 


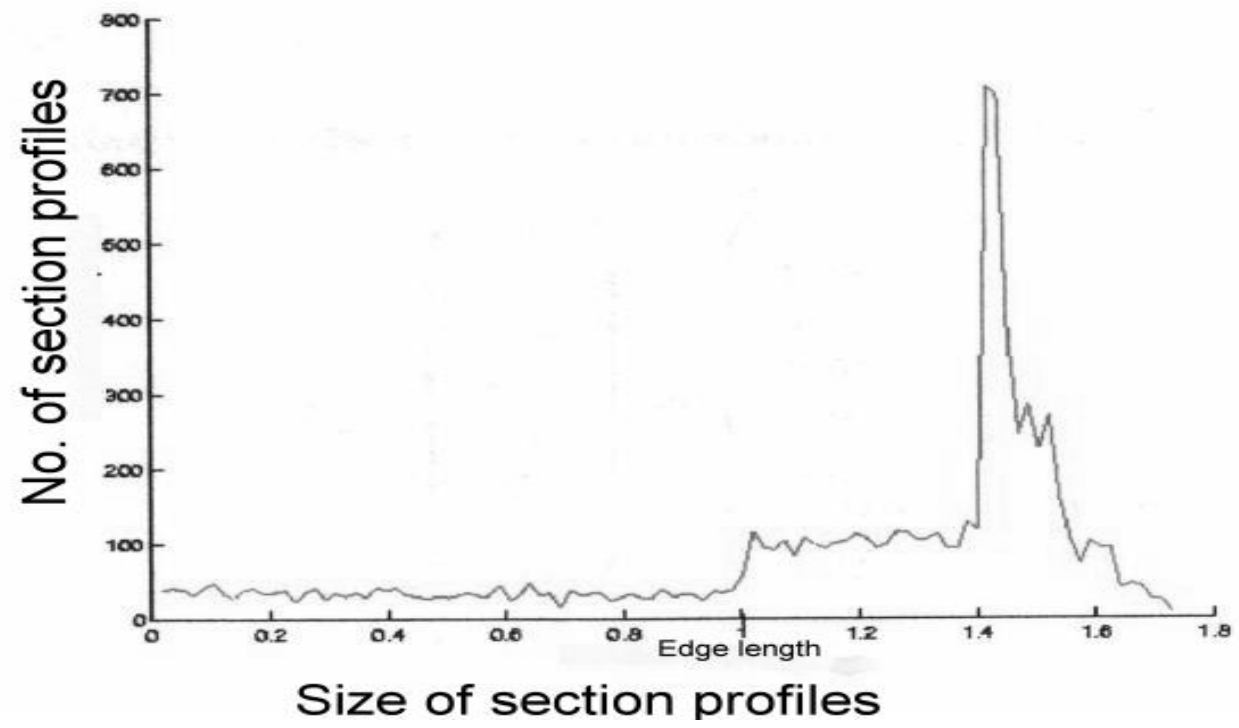

Figure 4.2: A frequency distribution of section profiles on simulated cross-section (from coarse grains).

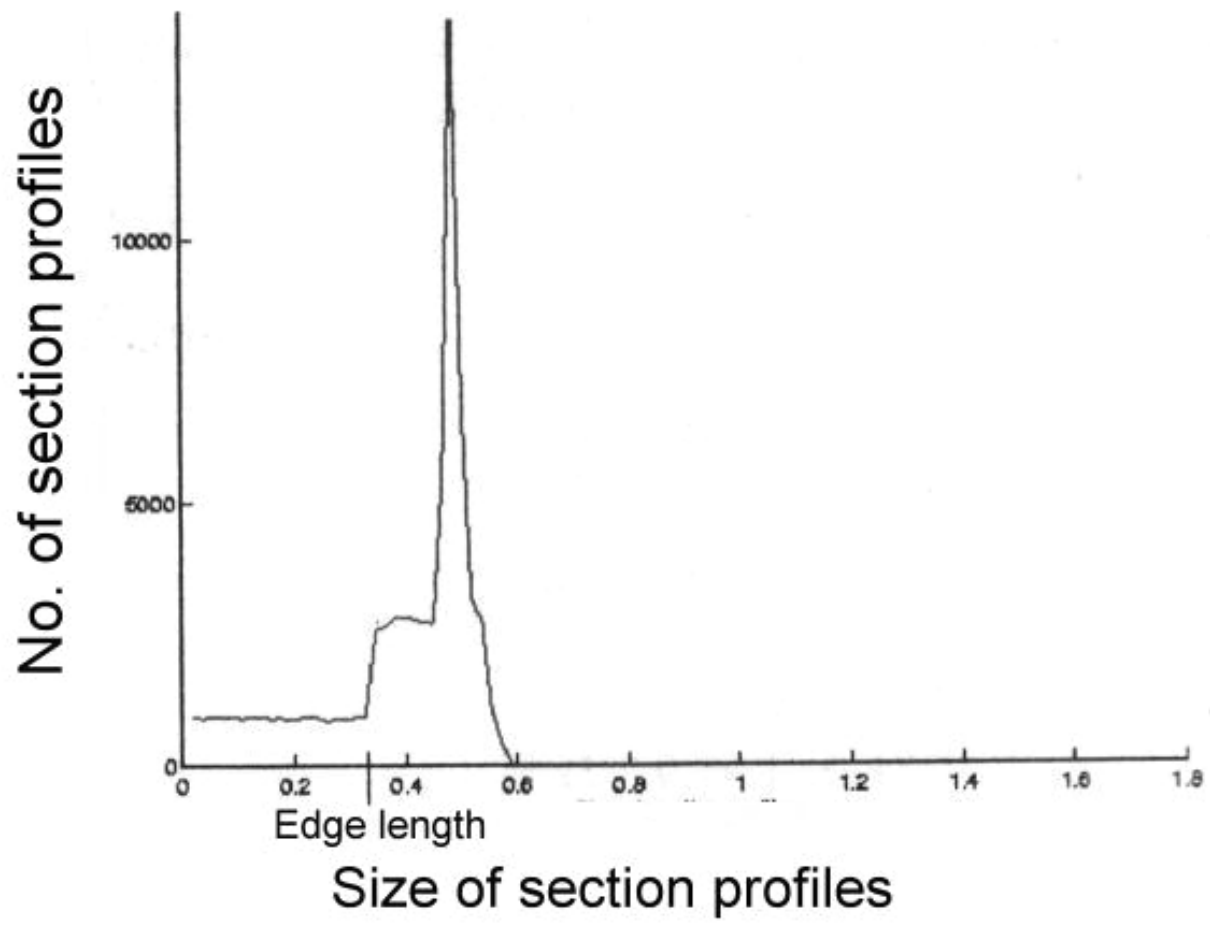

Figure 4.3: A frequency distribution of section profiles on simulated cross-section (from fine grains). 